\section{Is population genetics mired in the past?}

In a recent TREE perspective, Bossart and Prowell ${ }^{1}$ concluded that researchers estimating population structure used antiquated methods, and failed to acknowledge and test underlying model assumptions. We found these conclusions surprisingly pessimistic and decided to examine 67 papers on population structure from Evolution and Molecular Ecology (1997). Although many studies in Evolution were based solely on allozymes $(12 / 25)$, this was true of only $1 / 28$ papers in Molecular Ecology. The journal bias might explain some of Bossart and Prowell's findings, because they reviewed papers in Evolution that tended to focus primarily on theoretical problems in evolution, and only secondarily on estimating population structure.

We found neither that 'cautions [regarding analyses of population structure] have not been widely embraced by the scientific community', nor that 'conclusions often are drawn ... even though there are multiple, equally viable interpretations' 1 Only 14/67 papers calculated $\mathrm{Nm}$ (gene flow) and only two interpreted $\mathrm{Nm}$ literally. Most researchers viewed their results from a number of perspectives (e.g. historical association versus contemporary gene flow), and it was nearly impossible to find studies that did not use multiple loci and conduct sensitivity analyses over loci and/or populations.

Departures from equilibrium undoubtedly bias gene flow estimates in many cases. However, we disagree that allozymes yield no useful information regarding the movement of individuals. How can we test this assertion? Bossart and Prowell stated that comparisons with direct estimates of dispersal are "the only valid approach to the study and interpretation of gene flow in an ecological context'. We had difficulty interpreting this statement because it is well known that dispersal and gene flow are not equivalent for many reasons, and that rare dispersal events overlooked in most ecological studies can heavily influence indirect gene flow estimates ${ }^{2,3}$. Furthermore, there are other valid approaches for appraising gene flow estimates, such as examining correlations between $F_{\mathrm{st}}$ and morphological indicators of dispersal ability. It has been repeatedly shown that larval time is correlated with population differentiation in marine invertebrates ${ }^{4,5}$ and vertebrates ${ }^{6}$, flightless insects tend to have higher values of $F_{\text {st }}$ than flightform insects ${ }^{7-11}$, and $F_{\text {st }}$ values in plants are related to mode of seed dispersal ${ }^{12}$. These are not isolated examples; three separate reviews have found that wide dispersers tend to have higher estimates of $\mathrm{Nm}$ and lower estimates of $F_{\mathrm{st}}$ than those with restricted dispersal13-15. If ongoing gene flow has a negligible impact on allozyme differentiation among populations, there should be no correlation between population genetic differentiation and any measure of dispersal ability.

To conclude that population genetics is "no longer advancing [because of] our reliance on easy to apply, conventional indirect methods' implies that: (1) most evolutionary biologists use allozymes and F-statistics, and (2) this has stagnated the field. Yet the exclusive use of allozymes is becoming rarer and new statistical methodologies are published almost monthly. Therefore, we take a less pessimistic view than Bossart and Prowell. We believe that the limitations of traditional approaches are generally understood and that they still provide a valuable first approximation in many cases. Methods for determining the relative contributions of history and current gene flow are already being developed and utilized. Judging from the recent literature, we would argue that advancement, not stagnation, is the current state of the field.

\section{Andrew J. Bohonak \\ Neil Davies \\ George K. Roderick \\ Francis X. Villablanca}

\section{References}

1 Bossart, J.L. and Prowell, D.P. (1998) Trends Ecol. Evol. 13, 202-206

2 Peacock, M.M. (1997) Behav. Ecol. 8, 340-350

3 Kiester, A.R., Schwartz, C.W. and Schwartz, E.R. (1982) Evolution 36, 617-619

4 Hellberg, M.E. (1996) Evolution 50, 1167-1175

5 Hunt, A. (1993) Mar. Ecol. Prog. Ser. 92, 179-186

6 Waples, R.S. (1987) Evolution 41, 385-400

7 Liebherr, J.K. (1988) Evolution 42, 129-137

8 Preziosi, R.F. and Fairbairn, D.J. (1992) Evolution $46,430-444$

9 Varvio-Aho, S-L. and Pamilo, P. (1979) Hereditas 90, 237-249

10 Varvio-Aho, S-L. (1979) Hereditas 91, 207-214

11 Zera, A.J. (1981) Evolution 35, 218-225

12 Williams, C.F. and Guries, R.P. (1995) Evolution $48,791-805$

13 Peterson, M.A. and Denno, R.F. (1998) in Genetic Structure in Natural Insect Populations: Effects of Host Plants and Life History (Mopper, S. and Strauss, S.Y., eds), pp. 263-322, Chapman \& Hall

14 Bohonak, A.J. Q. Rev. Biol. (in press)

15 Govindaraju, D.R. (1988) Oikos 52, 31-35 